\title{
Low-temperature chemical synthesis of nanostructured indium nitride from indium hydroxide
}

\author{
Liwei FANG ${ }^{1}$, Liangbiao WANG ${ }^{1}{ }^{\dagger}$, Lei JIANG ${ }^{1}$, Ciyang ZHANG ${ }^{1}$, Tingting ZHOU $^{1}$, Zhe CHEN $^{1}$, Li LI ${ }^{1}$, \\ Xueping $\mathrm{ZHU}^{1}$, Yipeng $\mathrm{ZHU}^{1}$ and Tinghai YANG ${ }^{1,2, \ddagger}$ \\ ${ }^{1}$ School of Chemistry and Environment Engineering, Jiangsu University of Technology, Changzhou 213001, P. R. China \\ ${ }^{2}$ State Key Laboratory of Coordination Chemistry, Nanjing University, Nanjing 210093, P. R. China
}

\begin{abstract}
Nitridation of oxides is a conventional method to prepare nitrides. However, this method has a disadvantage of requiring a high reaction temperature. Additive-assisted nitridation is an effective route to synthesize nitrides at low temperature. In this study, hexagonal phase indium nitride (InN) nanomaterials have been successfully synthesized through the nitridation of indium hydroxide by sodium amide in a stainless-steel autoclave at a low temperature $\left(250-300^{\circ} \mathrm{C}\right)$, in which sodium thiosulfate acts as an additive. Scanning electron microscope image shows that the as-obtained InN sample is composed of nanoparticles and nanosheets. The influences of the reaction temperature on the as-obtained product have also been investigated.

(c)2020 The Ceramic Society of Japan. All rights reserved.
\end{abstract}

Key-words : Chemical synthesis, Nitrides, Low temperature, Semiconducting III-V materials, X-ray diffraction

[Received August 15, 2019; Accepted October 23, 2019]

Group IIIA nitrides (BN, AlN, GaN and $\mathrm{InN}$ ) are important semiconductors for their excellent properties such as high chemical inertness, radiation resistance, and high thermal conductivity. ${ }^{1), 2)}$ Among them, hexagonal phase indium nitride ( $\mathrm{InN}$ ) is a direct band-gap semiconductor with a narrow band-gap of about $0.70 \mathrm{eV}$, which has potential applications in photoelectronic devices. ${ }^{3)}$

Among group IIIA nitrides, the preparation and application of BN, AlN and GaN have been extensively studied. However, the decomposition temperature of $\mathrm{InN}$ is relatively low (about $500^{\circ} \mathrm{C}$ ), which makes the preparation of InN more difficult. ${ }^{4)}$ Because of the most challenging in production of $\mathrm{InN}$ nanomaterials, their properties and applications have not been well-investigated. Up to now, considerable efforts have been made in synthesizing nanostructured InN through different synthetic methods such as pyrolysis of $\mathrm{In}\left(\mathrm{NH}_{2}\right)_{3},{ }^{5)}$ organometallic precursor routes, ${ }^{6}$ ) high-pressure direct synthesis, ${ }^{7)}$ and high-temperature ammonolysis. ${ }^{8)}$ Takai et al. have synthesized InN thin films by reactive magnetron sputtering. ${ }^{9)}$ InN thin films can also be obtained through a metalorganic vapor phase epitaxy. ${ }^{10)}$ InN single crystals have been prepared by UVassisted atomic layer epitaxy. ${ }^{11)}$ Plasma-assisted atomic layer deposition can also be used to synthesize vertical III-nitride cylinder arrays. ${ }^{12)}$ Solvothermal synthesis of InN nanocrystalline has been reported in superheated

$\dagger$ Corresponding author: L. Wang; E-mail: lbwang@jsut. edu.cn

\$ Corresponding author: T. Yang; E-mail: tinghai_yang@ hotmail.com toluene. ${ }^{13)} \mathrm{Xu}$ et al. have synthesized InN nanowires and nanotubes by catalyst-free chemical vapor deposition at about $800^{\circ} \mathrm{C}^{14)} \mathrm{InN}$ nanowires with lengths of $30-50 \mu \mathrm{m}$ have been synthesized by nitriding of indium sesquioxide at $680-720^{\circ} \mathrm{C} .^{15)}$ Hsieh and his co-workers have synthesized $\mathrm{InN}$ nanocrystals with a diameter of $6 \mathrm{~nm}$ from $\mathrm{InBr}_{3}$ and $\mathrm{NaNH}_{2}$ at low temperature. ${ }^{16)}$ InN crystals can be obtained from the reaction of $\mathrm{LiInO}_{2}$ and $\mathrm{NaNH}_{2}$ at $240^{\circ} \mathrm{C} .{ }^{17), 18)}$ However, it is still a challenging task to synthesize InN through one-step chemical reaction at low temperature from cheap and rich raw materials.

Recently, an additive-assisted route has been reported to synthesized borides, nitrides, and carbides at a relatively low temperature in our research group. ${ }^{19)-25)}$ In this work, we report a new solid-state route to synthesize InN nanoparticles through a solid-state reaction of $\operatorname{In}(\mathrm{OH})_{3}, \mathrm{Na}_{2} \mathrm{~S}_{2} \mathrm{O}_{3}$ and $\mathrm{NaNH}_{2}$ at $250-300^{\circ} \mathrm{C}$. The synthetic method in this paper has the advantages of simple and feasible, low cost, high yield, and low reaction temperature.

The chemical reagents were used in the experiment without further purification: $\mathrm{Na}_{2} \mathrm{~S}_{2} \mathrm{O}_{3}$ (Sinopharm Chemical Reagent Co., Ltd., >99\%), $\mathrm{In}(\mathrm{OH})_{3}$ (Shanghai Macklin Biochemical Co., Ltd., 99.99\%), $\mathrm{NaNH}_{2}$ (Sinopharm Chemical Reagent Co., Ltd., >98\%), HCl (Chinasun Specialty Products Co., Ltd., analytical pure) and absolute ethyl alcohol (Sinopharm Chemical Reagent Co., Ltd., analytical pure). In a nitrogen-filled glovebox, $\mathrm{In}(\mathrm{OH})_{3}$ $(0.83 \mathrm{~g}), \mathrm{NaNH}_{2}(6.00 \mathrm{~g})$, and $\mathrm{Na}_{2} \mathrm{~S}_{2} \mathrm{O}_{3}(1.58 \mathrm{~g})$ were added into a stainless steel autoclave (about $22 \mathrm{~mL}$ capacity). Then, the autoclave was sealed tightly. The autoclave was heated to $300^{\circ} \mathrm{C}$ in a resistance furnace and maintained at 
$300^{\circ} \mathrm{C}$ for $10 \mathrm{~h}$. After cooling to room temperature, the autoclave was opened, and absolute ethyl alcohol was added in the autoclave. The black product was collected from the autoclave and washed with distilled water and diluted $\mathrm{HCl}$. The product was dried in vacuum oven at $60^{\circ} \mathrm{C}$ for $10 \mathrm{~h}$.

The as-obtained products were investigated by X-ray diffraction [XRD, Philips, $\mathrm{Cu} \mathrm{K} \alpha \lambda=1.54178 \AA$ ( $1 \AA=$ $0.1 \mathrm{~nm}$ )], field emission scanning electron microscope (FESEM, JEOL-JSM-6700F), transmission electron microscopy (JEOL-2010), X-ray photoelectron spectrometer (XPS, ESCALAB 250 spectrometer), and Raman spectrometer (Invia Raman spectrometer with an excitation laser wavelength of $514.5 \mathrm{~nm}$ ).

The crystal structure of the as-obtained product is determined from the XRD pattern (shown in Fig. 1). All the diffraction peaks in Fig. 1 can be indexed to (100), (002), (101), (102), (110), (103), (200), (112), (201), and (202) diffraction planes of the hexagonal phase $\mathrm{InN}$, respectively. The lattice parameters $a=3.5382 \AA$, and $c=5.7037 \AA$ have been extracted from the XRD pattern, which are close to the values of $a=3.5400 \AA$, and $c=5.7040 \AA$ (InN, JCPDS card No. 65-3412). Debye-Scherrer formula can also be used to estimate the crystalline size of the asobtained InN product. According to the Debye-Scherrer formula and the half-peak width of the InN (100) diffraction peak, the calculated size of the as-obtained $\operatorname{InN}$ product is about $48 \mathrm{~nm}$.

The morphologies of the as-obtained $\mathrm{InN}$ product are investigated by SEM and transmission electron microscope (TEM). From the SEM image [Fig. 2(a)], we found that the as-obtained $\mathrm{InN}$ product is composed of nanoparticles and hexagonal nanoplates (indicated by arrows).
The TEM image [Fig. 2(b)] shows the average size of the InN nanoparticles is about $50 \mathrm{~nm}$. Some fracted hexagonal InN nanoplates can also be found in the TEM image. A high-resolution TEM (HR-TEM) image of the as-obtained InN product is shown in Fig. 2(c). The regularly arranged lattice fringes have been found in Fig. 2(c), the distance is about $0.283 \mathrm{~nm}$, which corresponds to the (002) plane spacing hexagonal InN. All the above results prove that $\mathrm{InN}$ with high crystallinity has been synthesized through our designed chemical reaction. The energy dispersive Xray spectrometry (EDS) [shown in Fig. 2(d)] is employed for composition determination of the as-obtained $\mathrm{InN}$ product, which confirms that the as-obtained product consists of elements In and $\mathrm{N}$. The $\mathrm{Cu}$ peaks in the EDS is come from the TEM grid.

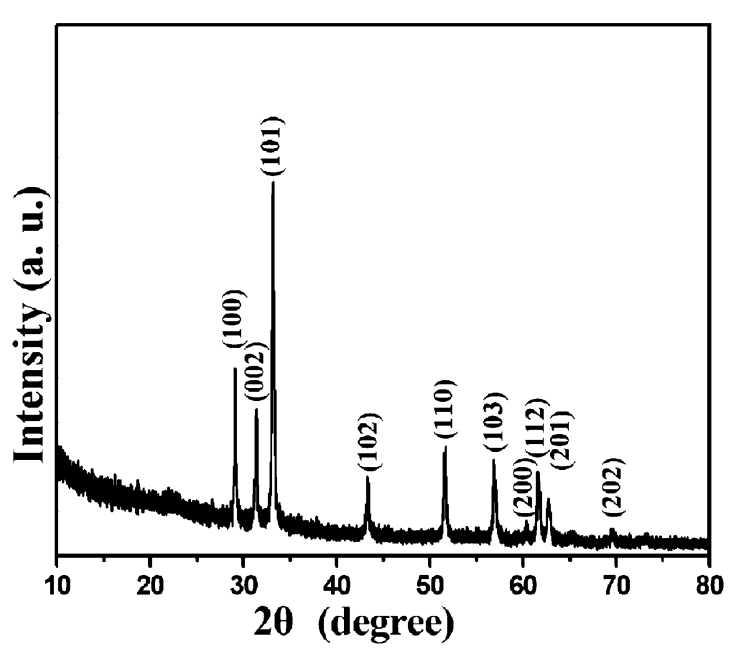

Fig. 1. XRD pattern of the as-obtained product.
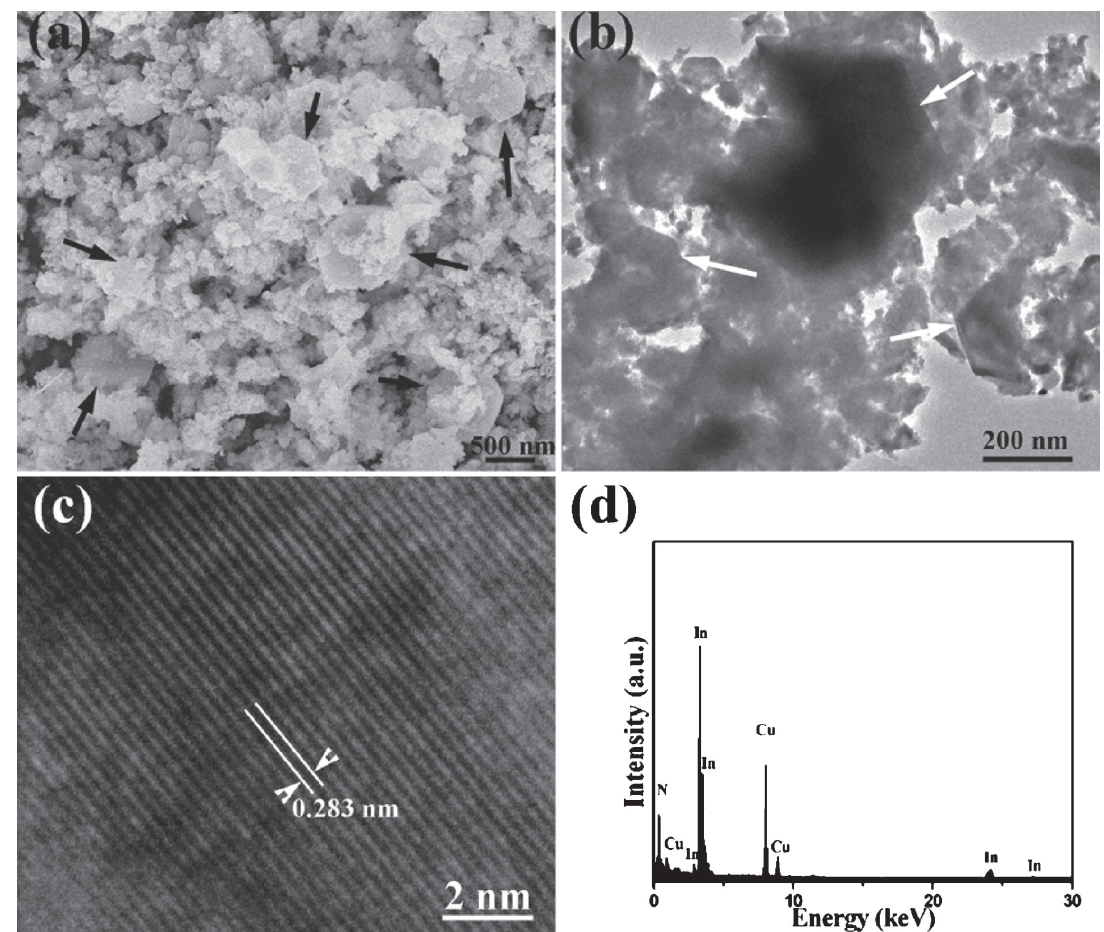

(d)

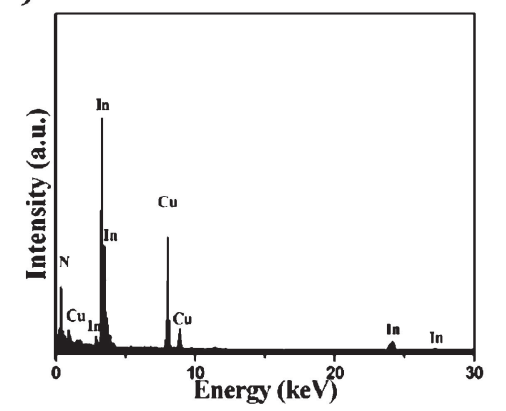

Fig. 2. FE-SEM image; (b) TEM image; (c) HR-TEM image of the InN product; (d) the corresponding EDS. 

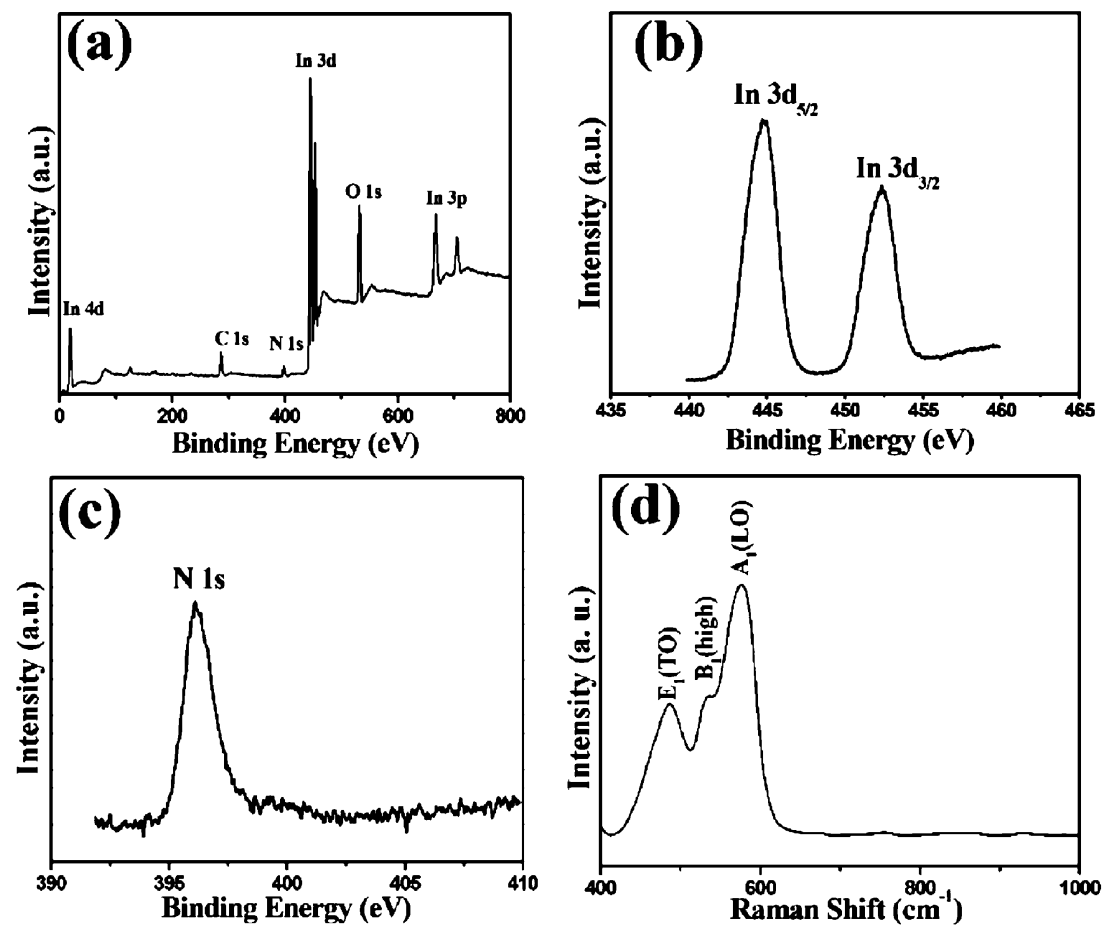

Fig. 3. XPS spectra of the InN product: (a) survey spectrum; (b) In3d region; (c) N1s region. (d) Raman spectrum of the as-obtained InN product.
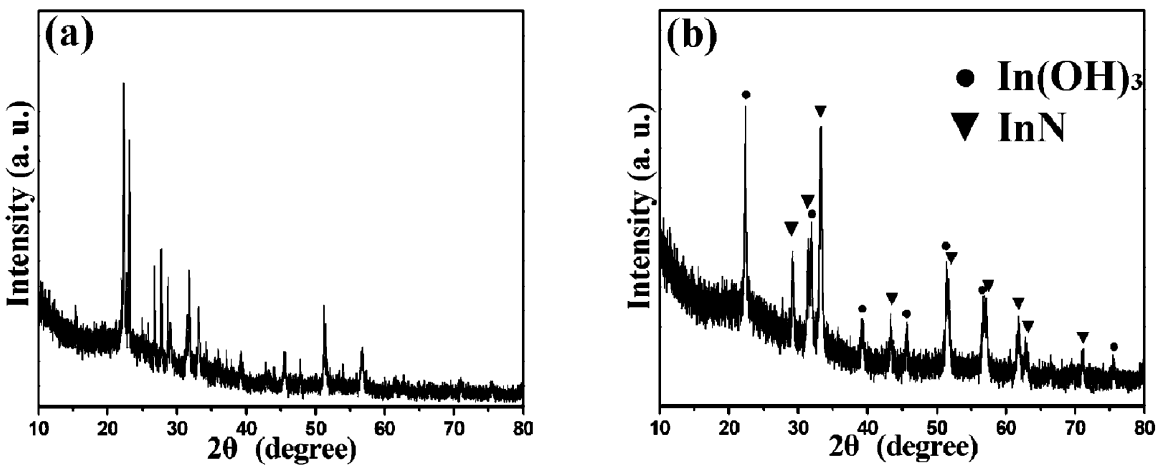

Fig. 4. $\mathrm{XRD}$ patterns of the products without $\mathrm{HCl}$ treatment obtained at different reaction temperatures: (a) $200^{\circ} \mathrm{C}$, (b) $250^{\circ} \mathrm{C}$.

The composition of the as-obtained InN product is investigated by XPS spectra. A typical survey spectrum of the InN product is shown in Fig. 3(a), which shows the product contains the elements In and $\mathrm{N}$. The appearance of other peaks is due to the absorption of $\mathrm{O}_{2}$ and $\mathrm{CO}_{2}$ impurities on the surface of the product. The two peaks in Fig. 3(b) centered at 444.78 and $452.36 \mathrm{eV}$ correspond to the $\operatorname{In} 3 \mathrm{~d}_{5 / 2}$ and $\operatorname{In} 3 \mathrm{~d}_{3 / 2}$ binding energies of $\mathrm{InN}$, respectively. The peak in Fig. 3(c) centered at $396.10 \mathrm{eV}$ corresponds to the N1s binding energy of InN. These results are close to the reported values for bulk InN. ${ }^{26)}$ A typical Raman spectrum of the as-obtained InN product is shown in Fig. 3(d). The three peaks centered at 486, 537, and 576 $\mathrm{cm}^{-1}$ correspond to the $\mathrm{E}_{1}(\mathrm{TO})$ mode, $\mathrm{B}_{1}$ (high) mode, and $\mathrm{A}_{1}(\mathrm{LO})$ mode of InN, respectively. The Raman spectrum is consistent with the result of the reported work, ${ }^{17)}$ which further proves that crystalline $\mathrm{InN}$ has been obtained through our synthetic route.

To investigate the effect of sodium thiosulfate in the formation process of $\mathrm{InN}$, we found that $\mathrm{InN}$ cannot be obtained from the reaction of $\mathrm{NaNH}_{2}$ and $\mathrm{In}(\mathrm{OH})_{3}$ through a similar experimental process. Therefore, sodium thiosulfate is an essential ingredient in the formation process of InN. In order to study the effect of reaction temperature on the product, some relevant experiments are carried out. From the XRD pattern of the as-obtained product, element sulfur is found in the obtained product at the reaction temperature of $200^{\circ} \mathrm{C}$ [Fig. 4(a)], which may be decomposed from sodium thiosulfate. When the reaction temperature is $250^{\circ} \mathrm{C}, \mathrm{InN}$ can also be obtained through this method [Fig. 4(b)], but the yield of $\mathrm{InN}$ is relatively low. The yield of InN can be improved by prolonging the 
reaction time at $250{ }^{\circ} \mathrm{C}$. The yield of $\mathrm{InN}$ is about $80 \%$ (according to the quality of indium hydroxide) at the reaction temperature of $300^{\circ} \mathrm{C}$ for $10 \mathrm{~h}$. In our synthetic experiments, neither expensive indium compounds nor a toxic ammonia flow is required. In addition, this synthesis method has the advantages of simple apparatus, low temperature, and high yield.

In summary, this work describes a novel thermal conversion route to InN nanomaterials from indium hydroxide. XRD pattern indicates that the product is hexagonal phase InN with the lattice parameters $a=3.5382 \AA$, and $c=5.7037 \AA$. Electron microscope images further reveal that the as-obtained InN product is composed of nanoparticles and hexagonal nanoplates. The influences of the reaction temperature on the as-obtained product have also been investigated. This simple method can also be applied to preparation of other metal nitrides.

Acknowledgements This work was supported by the College Students' Training Program of Innovation and Entrepreneurship of Jiangsu Province (no. 201911463044Y), Natural Science Foundation of Jiangsu Province (Grant no. BK20160292), National Natural Science Foundation of China (Grant no. 21701061 and 51872140), and the Changzhou Sci\&Tech Program (Grant no. CJ20179015).

\section{References}

1) H. Morkoc and S. N. Mohammad, Science, 267, 51-55 (1995).

2) D. A. Neumayer and J. G. Ekerdt, Chem. Mater, 8, 925 (1996)

3) T. Matsuoka, H. Okamoto, M. Nakao, H. Harima and E. Kurimoto, Appl. Phys. Lett., 81, 1246 (2002).

4) I. Akasaki and H. Amano, J. Cryst. Growth, 146, 455461 (1995).

5) A. P. Purdy, Inorg. Chem., 33, 282-286 (1994).

6) S. D. Dingman, N. P. Rath, P. D. Markowitz, P. C. Gibbons and W. E. Buhro, Angew. Chem. Int. Ed., 39, 1470-1472 (2000).

7) M. Bo'ckowski, Physica B, 265, 1-4 (1999).

8) P. Simek, D. Sedmidubsky, K. Klimova, S. Huber, P. Brazda, M. Mikulics, O. Jankovsky and Z. Sofer, J. Nanopart. Res., 16, 2805 (2014).
9) K. Ikuta, O. Takai and Y. Inoue, Thin Solid Films, 334, 49-53 (1998).

10) T. Akasaka, M. Schied and K. Kumakura, Appl. Phys. Express, 11, 081001 (2018).

11) T. Inushima, T. Shiraishi and V. Y. Davydov, Solid State Commun., 110, 491-495 (1999).

12) A. Haider, P. Deminskyi, M. Yilmaz, K. Elmabruk, I. Yilmaz and N. Biyikli, J. Mater. Chem. C, 6, 6471-6482 (2018).

13) J. Choi and E. G. Gillan, J. Mater. Chem., 16, 37743784 (2006).

14) H. Y. Xu, Z. Liu, X. T. Zhang and S. K. Hark, Appl. Phys. Lett., 90, 113105 (2007).

15) S. D. Luo, W. Y. Zhou, Z. X. Zhang, L. F. Liu, X. Y. Dou, J. X. Wang, X. W. Zhao, D. F. Liu, Y. Gao, L. Song, Y. J. Xiang, J. J. Zhou and S. S. Xie, Small, 1, 1004-1009 (2005).

16) J. C. Hsieh, D. S. Yun, E. Hu and A. M. Belcher, J. Mater. Chem., 20, 1435-1437 (2010).

17) A. Miura, T. Takei and N. Kumada, Cryst. Growth Des., 12, 4545-4547 (2012).

18) A. Miura, T. Takei and N. Kumada, J. Ceram. Soc. Jpn., 122, 86-88 (2014).

19) L. B. Wang, Q. L. Shen, D. J. Zhao, J. Lu, W. Q. Liu, J. H. Zhang, K. Y. Bao and Q. F. Zhou, J. Cryst. Growth, 471, 62-65 (2017).

20) K. L. Zhang, J. J. Lu, D. J. Zhao, L. B. Wang, H. F. Qin, W. Q. Liu and T. Mei, Chem. Lett., 47, 1318-1320 (2018).

21) L. B. Wang, Q. L. Shen, H. F. Qin, D. J. Zhao, W. Q. Liu, J. H. Sun, B. L. Zhu and Q. F. Zhou, J. Superhard Mater., 40, 392-394 (2018).

22) L. B. Wang, K. L. Zhang, H. L. Pan, L. Wang, D. Wang, W. C. Dai, H. F. Qin, G. R. Li and J. H. Zhang, Nanoscale, 10, 18936-18941 (2018).

23) L. B. Wang, Q. W. Li, Y. C. Zhu and Y. T. Qian, Int. J. Refract. Met. Hard Mater., 31, 288-292 (2012).

24) L. B. Wang, K. B. Tang, Y. C. Zhu, Q. W. Li, B. C. Zhu, L. C. Wang, L. L. Si and Y. T. Qian, J. Mater. Chem., 22, 14559-14564 (2012).

25) L. B. Wang, Y. X. Pan, Q. L. Shen, J. H. Zhang, K. Y. Bao, Z. S. Lou, D. J. Zhao and Q. F. Zhou, RSC $A d v$., 6, 98153-98156 (2016).

26) S. J. Patil, D. S. Bodas and A. B. Mandale, Appl. Surf. Sci., 245, 73-78 (2005). 